\title{
ACTIVITY OF SCAPULAR MUSCLES: COMPARISON OF OPEN AND CLOSED KINETIC CHAIN EXERCISES
}

\author{
Daina Šmite \\ Riga Stradins University, Department of Rehabilitation, Latvia \\ Irēna Upeniece \\ Riga Stradins University, Department of Sport and Nutrition, Latvia \\ Agnese Runce \\ Riga Stradins University, Department of Rehabilitation, Latvia \\ Helena Gapeyeva \\ University of Tartu, Institute of Sport Sciences and Physiotherapy, Estonia
}

\begin{abstract}
The function of the shoulder region and cervical spine are regulated by the motor control of the scapula. It is important to selectively activate weak muscles and minimally involve tense muscles to improve scapular motor control. The objective of this study was to compare the activity of scapular muscles and the intramuscular balance during various open and closed kinetic chain exercises. Methods: This study included 20 female sedentary office workers. A surface electromyography was used to analyze the activity of the scapular muscle in the correct exercise starting position and during 6 different exercises. Additionally, the optimal intramuscular balance was examined. Results and conclusions: Open kinetic chain exercises is more suitable than closed kinetic chain exercises for training scapular active stability. Horizontal shoulder abduction with external rotation (scapula retraction and internal rotation) while in the prone position was optimal and could be recommended (as well as its modifications with a resistance band) for training programs to improve scapula active stability.
\end{abstract}

Keywords: exercises, muscle activity, motor control, scapula, stability.

\section{Introduction}

Proper motor control of the scapula is essential to the function of the shoulder region and the cervical spine. The functions of the scapular muscles and their disorders are frequently studied, and there is evidence that altered kinematics of shoulder blades are observed in individuals with subacromial impingement syndrome (Timmons et al., 2012).

Scapular motor control is related to the cervical spine, which can be found in the study by Gaffney et al. (2014), who evaluated the relationship between the 
Daina Šmite, Irēna Upeniece, Agnese Runce, Helena Gapeyeva. Activity of Scapular Muscles: Comparison of Open and Closed Kinetic Chain Exercises

activation of the trapezius muscle and both the scapular and cervical posture. Taking into account the wide-spread neck pain found in office workers, the research of Park \& Yoo (2013) identified alterations of the scapula position after continuous work with the computer keyboard.

It is important to selectively activate weak muscles and their parts with minimal participation of tense muscles to improve scapular motor control during exercises. In the open kinematic chain, it is easier to activate a certain muscle separately, which is impossible in the closed kinematic chain. The purpose of the closed kinematic chain is to improve stability (Kisner \& Colby, 2007).

Previous studies examining the functions of scapular muscles analyzed athletes with musculoskeletal disorders of the shoulder region (Ratcliffe et al., 2014; Timmons et al., 2012; Cools et al., 2007; De Mey et al., 2009a, 2013b, $2014 \mathrm{c}$ ). There is a relationship between the motor control of the scapula, the function of cervical spine, and pain in the neck and upper back. Thus, it is useful to determine the most optimal exercises to improve scapula motor control in clinical practice and health promotion programs for target groups such as office workers.

The objective of this study was to compare the activity of scapular muscles and the intramuscular balance during various open and closed kinetic chain exercises (OKCE, CKCE).

\section{Methods}

This study examined 20 female sedentary office workers from one establishment who were not involved in sports activities on a daily basis. The mean participant age was $42.3 \pm 9.9$ years. The exclusion criteria included the following: actual pain in the cervical spine and/or the shoulder region, injury and/or surgery of the cervical spine or the shoulder region, arthrosis of the shoulder joint, congenital pathology of the spine, red flag signs of the cervical spine, and malignancies.

The study was performed as a cross-sectional analysis. Each participant was measured on Monday morning (after two resting days, before work). The approval for this study was obtained from the Ethics Committee of Riga Stradins University, and each subject was required to sign a consent form prior to study participation.

Surface electromyography (sEMG) was used to register the activity of muscles during the analyzed exercises. sEMG records were performed with ME 6000 biomonitor (Mega electronics Ltd, Finland) and analysed by the MegaWin 3.0 Software (Mega electronics Ltd, Finland). Noraxon Dual electrodes (Naroxon U.S.A. Inc., Scottsdale, Arizona, USA) were used. The location of 
electrodes to each muscle was based on the clinical sEMG protocols by Sella (2000).

The sEMG was recorded during each exercise by using the time intervals for correct starting position (muscle pre-activation) and during exercise performance. The mean electromyographic activity $(\mu \mathrm{V})$ was normalized by the maximal voluntary isometric contraction (\% of MVC) and was analyzed across $3 \mathrm{OKCE}$ and $3 \mathrm{CKCE}$. The MVC was obtained during isometric strength testing of each muscle (Kendall et al, 1993). Two main local stability muscles (the serratus anterior (SA) and the lower trapezius (LT)) and global mobility muscles (the levator scapulae (LS) and the pectoralis minor (PM)) were chosen for the analysis. The intramuscular balance is reached when the activity of local stability muscles is higher than the activity of global mobility muscles. All analyzed exercises were performed in low positions and with low intensity.

Each participant had to perform six therapeutic exercises. The closed kinematic chain included the following exercises: transfer of weight in forearm support (No. 1), transfer of weight on all fours (No. 2), and transfer of weight with arm support against a wall (90 degrees flexion in shoulders) (No. 3). The open kinematic chain included the following exercises: reaching upwards (scapula protraction and external rotation) while in the supine position with 90 degrees flexion in shoulders (No. 4), horizontal shoulder abduction with external rotation (scapula retraction and internal rotation) while prone (No. 5), and reaching forward (scapula protraction and external rotation) in a sitting position with 90 degrees flexion in shoulders (No. 6).

Each exercise was performed twice. The first exercise was a trial used for correction (explanation and demonstration was performed before). The second repetition was used to collect measurements. The three OKCE (sitting, supine, prone lying) were performed first (with 10 minutes rest between each exercise). The participants then had 30 minutes rest before the three CKCE (standing, forearm, all fours) exercises were performed (with 10 minutes rest between each exercise).

The results were analyzed using SPSS.V20 software. We used the mean and standard deviation to describe the results for each muscle and exercise. A paired t-test was used to calculate the difference between mean values of local stability muscles (SA, LT) for EMG activity. The statistically significant level was $\mathrm{p}<0.05$.

\section{Results}

The highest pre-activity SA occurred for OKCE No. $4(\mathrm{p}<0.001)$. The SA pre-activity in all OKCE were higher $(\mathrm{p}<0.05)$ than all CKCE. The highest LT 
Daina Šmite, Irēna Upeniece, Agnese Runce, Helena Gapeyeva. Activity of Scapular Muscles: Comparison of Open and Closed Kinetic Chain Exercises

pre-activity was found for OKCE No. $5(\mathrm{p}<0.05)$. The other two OKCE demonstrated higher LT pre-activity than CKCE No. 1 and No. $2(\mathrm{p}<0.05)$.

The highest SA and LT activity occurred during exercise OKCE No. 5 $(\mathrm{p}<0.05)$. In the other OKCE (No. 4 and 6), the SA activity was higher than in the two CKCE (No. 1 and 2) and was equal in CKCE No. $3(p<0.05)$. The second highest LT activity was observed during CKCE No. $3(\mathrm{p}<0.05)$. The other CKCE (No. 1 and 2) had less LT activity than the two OKCE (No. 5 and 6). However, the LT activity was equal to OKCE No. 4 (p<0.05) ("Fig. 1" and "Fig. 2").

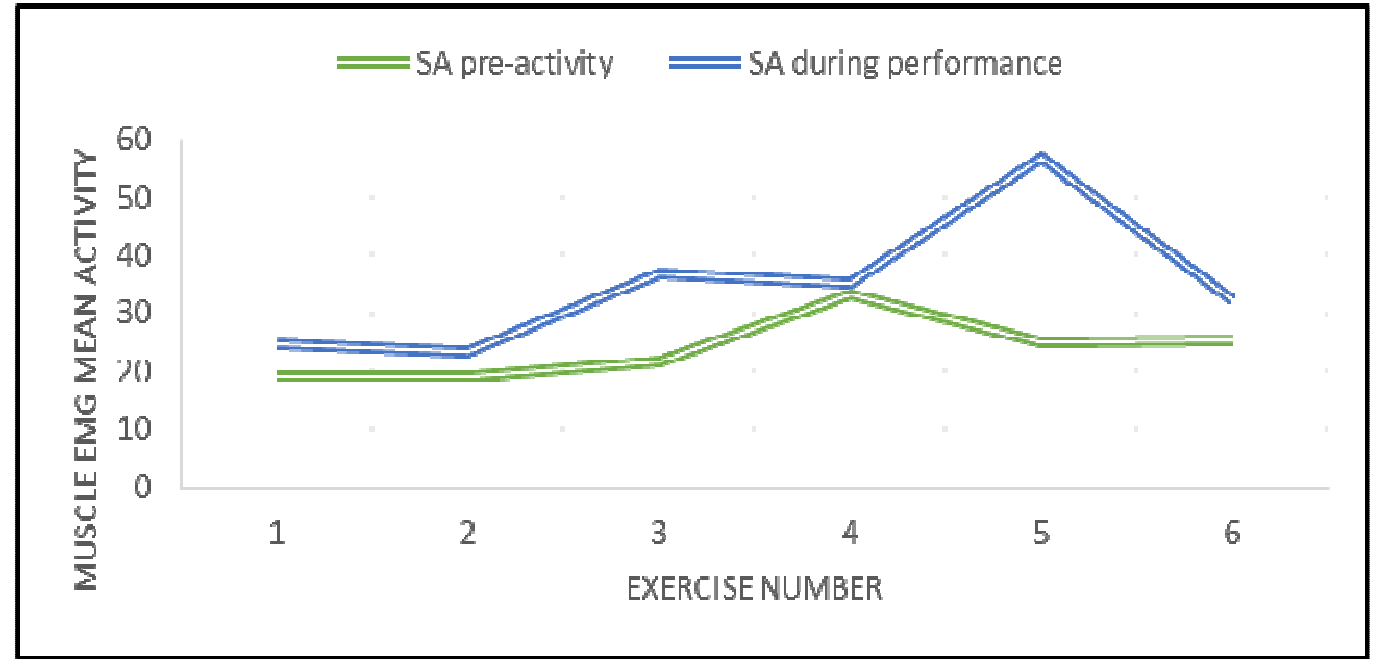

Fig.1 SA mean activity (\% of MVC) in analysed OKCE and CKCE

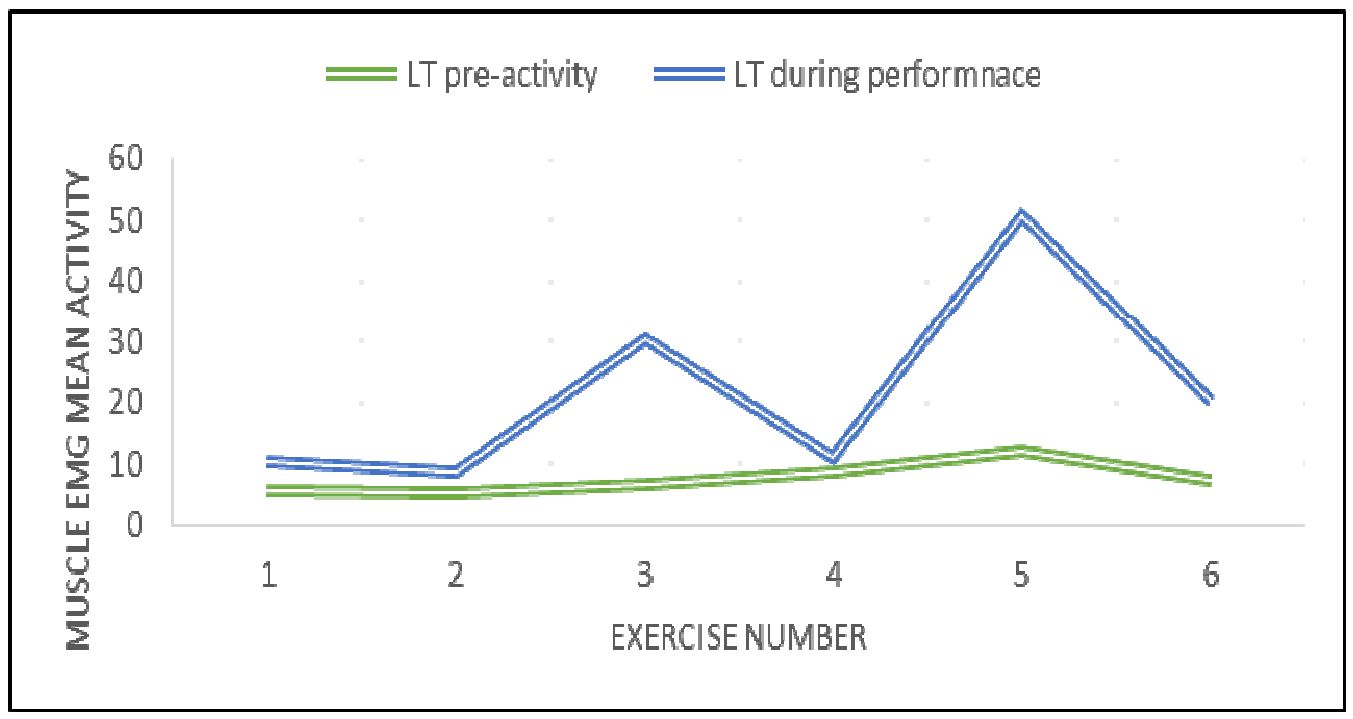

Fig.2 LT mean activity (\% of MVC) in analysed OKCE and CKCE 
Intramuscular balance: The activity of both analyzed local stability muscles was higher than the activity of both analyzed mobility muscles in OKCE No. 5.

The SA/LS balance was optimal in all the OKCE and the two CKCE (No. 1 and 3). The SA/PM balance was optimal in the two OKCE (No. 4 and 5) (in OKCE No. 6, SA and PM activity was equal) and in the one CKCE (No. 1) (in CKCE No. 2 and 3, PM activity was greater than SA activity). The LT/LS balance was optimal in the two OKCE (No. 5 and 6) and in CKCE No. 3 (in the remaining exercises, LS activity was greater than LT). The LT/PM relationship was optimal in OKCE No. 5.

\section{Discussion}

The main findings of our study were the following: (1) all three analyzed OKCE demonstrated higher SA activity during correct start position than CKCE, and the LT was more pronounced in the start position of OKCE than CKCE, with the exception of one CKCE (No. 3); (2) the highest SA and LT activity was demonstrated during performance of OKCE No. 5, which is the horizontal shoulder abduction with external rotation (scapula retraction and internal rotation) while prone; (3) the SA and LT activity during exercises does not suggest the superiority of analyzed OKCE or CKCE; (4) analyzed OKCE demonstrated more optimal intramuscular balance with higher SA and LT activity and less LS and PM activity.

The results of our research indicate that the involvement of SA in the start position was sufficient (i.e., at least $20 \% \mathrm{MVC}$ ) in all analyzed exercises. However, the LT did not reach $20 \%$ of MVC in any of the exercises. Thus, it can be assumed that there was insufficient control of the scapula during elevation (taking into account that simultaneous scapular kinematic analysis was not performed, and it cannot be either confirmed or denied). The data suggest it is necessary to reinforce the involvement of the LT at the start position of these exercises.

There was more involvement of stability muscles for the analyzed OKCE in correct exercise start positions than CKCE. Therefore, training with the correct start position for the analyzed OKCE is an option for scapula motor control improvement.

The SA and LT activity during exercise did not show the superiority of analyzed OKCE or CKCE despite evidence $(p<0,05)$ that the highest SA and LT activity was demonstrated during OKCE No. 5, which is the horizontal shoulder abduction with external rotation (scapula retraction and internal rotation) while prone. This was the only exercise that showed optimal intramuscular balance for all analyzed muscles. The study of Cools et al., (2007) demonstrated the relationship is optimal between the upper trapezius and LT for this exercise (as 
Daina Šmite, Irēna Upeniece, Agnese Runce, Helena Gapeyeva. Activity of Scapular Muscles: Comparison of Open and Closed Kinetic Chain Exercises

well as the relationship between the upper and the middle trapezius). However, the relationship is not optimal for the upper trapezius and SA.

The results of our research suggest that improving the active stability of the scapula is best achieved using OKCE No. 5. However, the start position of this exercise requires additional attention regarding the LT. Clinical practice and health promotion programs use resistance band exercises to produce the same shoulder movements (horizontal abduction from 90 degrees flexion with external rotation) and scapula movement (retraction and internal rotation). The major difference is that the starting position can be lying on the back, sitting or standing. These exercises could be used to improve the motor control of the scapula in case a prone position is contraindicated or if the power of involved muscles is insufficient to overcome gravity or to diversify the program of exercises. By considering the cervical spine position in exercises while prone (which are less analyzed in research literature) and while lying on the back or sitting/standing, it is possible that the activity of the upper trapezius will change and could form an optimal relationship with SA. However, this possibility requires additional investigation and was not the objective of this research.

The results show that the transfer of weight using forearm support used the least mobility muscles and was equal to the activity of both stability muscles (also relatively low). However, using facilitation methods for stability muscles (especially LT) will allow this exercise to be effectively integrated into an exercise program.

There was excessive activity of mobility muscles and relatively low activity of stability muscles observed in the transfer of weight on all fours and while reaching upwards (scapula protraction and external rotation) while supine with 90 degree shoulder flexion. The position on all fours is frequently used in practice to improve active stability of the scapula. The results of our research show that despite high SA activity, the LT is not sufficiently involved and the activity of mobility muscles is excessively in this position. Therefore, this posture and more complicated variations of this posture (unstable support surface that can reduce the activity of SA (Kalantari \& Ardestani, 2014)) are not recommended for improving scapula stability. The position on all fours also requires sufficient control of the lower part of the body (the lumbar spine, pelvis, hips and knees). These aspects were not analyzed in this study relative to the scapula activity (also lacking in the literature). The exercise that involves reaching upwards (scapula protraction and external rotation) while supine with 90 degree shoulder flexion is part of a clinical SA training program. However, the excessive activity of mobility muscles (especially PM) does not indicate this is an optimal exercise.

The study cohort was sufficiently homogeneous (age, gender, functional status, and daily risk factors), which allowed the data to be properly analyzed 
and interpreted. The surface EMG was the chosen assessment method in this study. EMG is adequately informative and provided the expected results. The EMG normalization process is one study limitation because determining the maximal voluntary contraction activity is complicated and there is no unified protocol for scapular muscles. Therefore, the muscle power tests were used as a reference. Future research studies should use several tests for each muscle. The muscle activity results are another limitation to this study. Although the exercises were practiced before the measurements were recorded, the precision of their execution was low. However, the results are similar to actual situations because these exercises are frequently utilized in group sessions or training programs at home where individual correction and guidance is impossible.

\section{Conclusions}

- Open kinetic chain exercises are more suitable than closed kinetic chain exercises for training scapular active stability.

- Horizontal shoulder abduction with external rotation (scapula retraction and internal rotation) while in the prone position was optimal and could be recommended (as well as its modifications with a resistance band) for training programs to improve scapula active stability. As well this exercise is suitable for SA strength training based on results of previous studies.

\section{References}

Cools, A. M., Declercq, G. A., Cambier, D. C., Mahieu, N. N., \& Witvrouw, E. E. (2007). Trapezius activity and intramuscular balance during isokinetic exercise in overhead athletes with impingement symptoms. Scand J Med Sci Sports, 17, 25-33

De Mey, K., Cagnie, B., Danneels, L. A., Cools, A. M., \& Van de Velde, A. (2009). Trapezius muscle timing during selected shoulder rehabilitation exercises. J Orthop Sports Phys Ther, 39, 743-52

De Mey, K., Danneels, L., Cagnie, B., Borms, D., T’Jonck, Z., Van Damme, E., \& Cools A. M. (2014). Shoulder muscle activation levels during four closed kinetic chain exercises with and without Redcord slings. J Strength Cond Res, 28 (6), 1626-35

De Mey, K., Danneels, L., Cagnie, B., Van den Bosch, L., Flier, J., \& Cools, A. M. (2013). Kinetic chain influences on upper and lower trapezius muscle activation during eight variations of a scapular retraction exercise in overhead athletes. J Sci Med Sport,16, 6570

Gaffney, B. M., Maluf, K. S., Curran-Everett, D., \& Davidson, B. S. (2014). Associations between cervical and scapular posture and the spatial distribution of trapezius muscle activity. J Electromyogr Kinesiol, 24 (4), 542-9.

Kalantari, K. K., \& Ardestani, B. S. (2014). The effect of base of support stability on shoulder muscle activity during closed kinematic chain exercises. Journal of Bodywork and Movement Therapies, 18 (2), 233-8. 
Daina Šmite, Irēna Upeniece, Agnese Runce, Helena Gapeyeva. Activity of Scapular Muscles: Comparison of Open and Closed Kinetic Chain Exercises

Kendall, F. P., McCreary, E. K., \& Provance, P. G. (1993). Muscles testing and function. Williams and Wilkins. Baltimore.

Kisner, C., \& Colby, L. A. (2007). Therapeutic exercise: Foundations and Techniques. Philadelphia: Davis Company.

Park, S. Y., \& Yoo, W. G. (2013). Effect of sustained typing work on changes in scapular position, pressure pain sensitivity and upper trapezius activity. J Occup Health, 55 (3), 167-72.

Ratcliffe, E., Pickering, S., McLean, S., \& Lewis, J. (2014). Is there a relationship between subacromial impingement syndrome and scapular orientation? A systematic review. $\mathrm{Br}$ J Sports Med, 48 (16), 1251-6.

Sella, G. E. (2000). Guidelines for neuromuscular re-education with sEMG biofeedback. Volume I. GENMED Publishing, Martins Ferry, OH, USA.

Timmons, M. K., Thigpen, C. A., Seitz, A. L., Karduna, A. R., Arnold, B. L., \& Michener, L. A. (2012). Scapular kinematics and subacromial-impingement syndrome: a metaanalysis. J Sport Rehabili, 21 (4), 354-70. 


\section{INFORMĀCIJAS TEHNOLOĢIJU IZMANTOŠANA IZGLİTİBĀ INFORMATION TECHNOLOGIES IN EDUCATION}


